\title{
Entropy production fluctuation theorem and the nonequilibrium work relation for free energy differences
}

\author{
Gavin E. Crooks* \\ Department of Chemistry, University of California at Berkeley, Berkeley, California 94720
}

(Received 17 February 1999)

\begin{abstract}
There are only a very few known relations in statistical dynamics that are valid for systems driven arbitrarily far-from-equilibrium. One of these is the fluctuation theorem, which places conditions on the entropy production probability distribution of nonequilibrium systems. Another recently discovered far from equilibrium expression relates nonequilibrium measurements of the work done on a system to equilibrium free energy differences. In this paper, we derive a generalized version of the fluctuation theorem for stochastic, microscopically reversible dynamics. Invoking this generalized theorem provides a succinct proof of the nonequilibrium work relation. [S1063-651X(99)10109-0]
\end{abstract}

PACS number(s): 05.70.Ln, 47.52.+j, 82.20.Mj

\section{INTRODUCTION}

Consider some finite classical system coupled to a constant temperature heat bath, and driven out of equilibrium by some time-dependent work process. Most relations of nonequilibrium statistical dynamics that are applicable to systems of this sort are valid only in the linear, near-equilibrium regime. One group of exceptions is the entropy production fluctuation theorems [1-12], which are valid for systems perturbed arbitrarily far away from equilibrium. Although the type of system, range of applicability, and exact interpretation differ, these theorems have the same general form,

$$
\frac{P(+\sigma)}{P(-\sigma)} \simeq e^{\tau \sigma} .
$$

Here $P(+\sigma)$ is the probability of observing an entropy production rate, $\sigma$, measured over a trajectory of time $\tau$. Evans and Searles [2] gave a derivation for driven thermostated deterministic systems that are initially in equilibrium, Gallavotti and Cohen [3] rigorously derived their fluctuation theorem for thermostated deterministic steady-state ensembles, and Kurchan [9], Lebowitz and Spohn [11], and Maes [12] have considered systems with stochastic dynamics. The exact interrelation between these results is currently under debate $[13,14]$.

In this paper we will derive the following, somewhat generalized, version of this theorem for stochastic microscopically reversible dynamics:

$$
\frac{P_{\mathrm{F}}(+\omega)}{P_{\mathrm{R}}(-\omega)}=e^{+\omega} .
$$

Here $\omega$ is the entropy production of the driven system measured over some time interval, $P_{\mathrm{F}}(\omega)$ is the probability distribution of this entropy production, and $P_{\mathrm{R}}(\omega)$ is the probability distribution of the entropy production when the system is driven in a time-reversed manner. This distinction is necessary because we will consider systems driven by a

*Electronic address: gavinc@garnet.berkeley.edu time-dependent process, rather than the steady perturbation considered elsewhere. The use of an entropy production, rather than an entropy production rate, will prove convenient.

As a concrete example of a system for which the above theorem is valid, consider a classical gas confined in a cylinder by a movable piston. The walls of this cylinder are diathermal so that the gas is in thermal contact with the surroundings, which therefore act as the constant temperature heat bath. The gas is initially in equilibrium with a fixed piston. The piston is then moved inwards at a uniform rate, compressing the gas to some new, smaller volume. In the corresponding time-reversed process, the gas starts in equilibrium at the final volume of the forward process, and is then expanded back to the original volume at the same rate that it was compressed by the forward process. The microscopic dynamics of the system will differ for each repetition of this process, as will the entropy production, the heat transfer, and the work performed on the system. The probability distribution of the entropy production is measured over the ensemble of repetitions.

Another expression that is valid in the far-fromequilibrium regime is the recently discovered relationship between the difference in free energies of two equilibrium ensembles, $\Delta F$, and the amount of work, $W$, expended in switching between ensembles in a finite amount of time [1519],

$$
\left\langle e^{-\beta W}\right\rangle=e^{-\beta \Delta F}
$$

Here $\beta=1 / k_{B} T, k_{B}$ is the Boltzmann constant, $T$ is the temperature of the heat bath that is coupled to the system, and \langle\rangle indicates an average over many repetitions of the switching process. For the confined gas considered above, the free energy depends on the position of the piston. With Eq. (3), we can calculate the free energy change when the system is compressed to a new volume by making many measurements of the work required to effect the change, starting each time from an equilibrated system, and taking the above average. In the limit of instantaneous switching between ensembles, this relation is equivalent to the standard thermodynamic 
perturbation method used to calculate free energy differences with computer simulations [20-23].

Equations (2) and (3) are actually closely related. We first note that whenever Eq. (2) is valid, the following useful relation holds [see Eq. (16) of Ref. [8]]:

$$
\left\langle e^{-\omega}\right\rangle=\int_{-\infty}^{+\infty} P_{\mathrm{F}}(+\omega) e^{-\omega} d \omega=\int_{-\infty}^{+\infty} P_{\mathrm{R}}(-\omega) d \omega=1 .
$$

We shall show in Sec. III that the generalized fluctuation theorem, Eq. (2), can be applied to systems that start in equilibrium, and that the entropy production $\omega$ for such systems is $-\beta \Delta F+\beta W$. The nonequilibrium work relation for the free energy change, Eq. (3), can thus be derived by substituting this definition of the entropy production into Eq. (4), and noting that the free energy difference is a state function, and can be moved outside the average.

In the following section we will derive the fluctuation theorem, Eq. (2). Then in Sec. III we will discuss two distinct groups of driven systems for which the theorem is valid. In the first group, systems start in equilibrium, and are then actively perturbed away from equilibrium for a finite amount of time. In the second group, systems are driven into a time symmetric nonequilibrium steady state. We conclude by discussing several approximations that are valid when the entropy production is measured over long time periods. The fluctuation theorem and its approximations are illustrated with data from a simple computer model.

\section{THE FLUCTUATION THEOREM}

As indicated in the Introduction, we consider finite, classical systems coupled to a heat bath of constant temperature, $T=1 / \beta$. (All entropies are measured in nats [24], so that Boltzmann's constant is unity.) The state of the system is specified by $x$ and $\lambda$, where $x$ represents all the dynamical, uncontrolled degrees of freedom, and $\lambda$ is a controlled, timedependent parameter. For the confined gas considered in the Introduction, the heat bath is simply the walls of the cylinder, the state vector $x$ specifies the positions and momenta of all the particles, and $\lambda$ specifies the current position of the piston. In computer simulations, the controlled parameter could be a microscopic degree of freedom. For example, in a free energy calculation $\lambda$ could specify the distance between two particles or the chemical identity of an atom or molecule [20-23].

A particular path through phase space will be specified by the pair of functions $(x(t), \lambda(t))$. It will prove convenient to shift the time origin so that the paths under consideration extend an equal time on either side of that origin, $t \in\{-\tau$, $+\tau\}$ or $t \in\{-\infty,+\infty\}$. Then the corresponding timereversed path can be denoted as $(\bar{x}(-t), \bar{\lambda}(-t))$. The overbar indicates that quantities odd under a time reversal (such as momenta or an external magnetic field) have also changed sign.

The dynamics of the system are required to be stochastic and Markovian [25]. The fluctuation theorem was originally derived for thermostated, reversible, deterministic systems $[2,3]$. However, there are fewer technical difficulties if we simply assume stochastic dynamics [9]. We will also require that the dynamics satisfy the following microscopically reversible condition:

$$
\frac{\mathcal{P}[x(+t) \mid \lambda(+t)]}{\mathcal{P}[\bar{x}(-t) \mid \bar{\lambda}(-t)]}=\exp \{-\beta Q[x(+t), \lambda(+t)]\} .
$$

Here $\mathcal{P}[x(+t) \mid \lambda(+t)]$ is the probability, given $\lambda(t)$, of following the path $x(t)$ through phase space, and $\mathcal{P}[\bar{x}$ $(-t), \mid \bar{\lambda}(-t)]$ is the probability of the corresponding timereversed path. $Q$ is the heat, the amount of energy transferred to the system from the bath. The heat is a functional of the path, and odd under a time reversal, i.e., $Q[x(t), \lambda(t)]=$ $-Q[\bar{x}(-t), \bar{\lambda}(-t)]$.

In current usage, the terms "microscopically reversible", and "detailed balance" are often used interchangeably [26]. However, the original meaning of microscopic reversibility $[27,28]$ is similar to Eq. (5). It relates the probability of a particular path to its reverse. This is distinct from the principle of detailed balance [26,21], which refers to the probabilities of changing states without reference to a particular path. It is the condition that $P(A \rightarrow B)=P(B \rightarrow A) \exp$ $(-\beta \Delta E)$, where $\Delta E$ is the difference in energy between state $A$ and state $B$, and $P(A \rightarrow B)$ is the probability of moving from state $A$ to state $B$ during some finite time interval.

The stochastic dynamics that are typically used to model reversible physical systems coupled to a heat bath, such as the Langevin equation and Metropolis Monte Carlo, are microscopically reversible in the sense of Eq. (5). Generally, if the dynamics of a system are detail balanced locally in time (i.e., each time step is detail balanced), then the system is microscopically reversible even if the system is driven from equilibrium by an external perturbation [see Eq. (9) of Ref. [19]].

A particular work process is defined by the phase-space distribution at time $-\tau, \rho\left(x_{-\tau}\right)$, and the value of the control parameter as a function of time, $\lambda(t)$. Each individual realization of this process is characterized by the path that the system follows through phase space, $x(t)$. The entropy production, $\omega$, must be a functional of this path. Clearly there is a change in entropy due to the exchange of energy with the bath. If $Q$ is the amount of energy that flows out of the bath and into the system, then the entropy of the bath must change by $-\beta Q$. There is also a change in entropy associated with the change in the microscopic state of the system. From an information theoretic [24] perspective, the entropy of a microscopic state of a system, $s(x)=-\ln \rho(x)$, is the amount of information required to describe that state given that the state occurs with probability $\rho(x)$. The entropy of this (possibly nonequilibrium) ensemble of systems is $S=-\Sigma_{x} \rho(x) \ln \rho(x)$. Thus, for a single realization of a process that takes some initial probability distribution, $\rho\left(x_{-\tau}\right)$, and changes it to some different final distribution, $\rho\left(x_{+}\right)$, the entropy production [29] is

$$
\omega=\ln \rho\left(x_{-\tau}\right)-\ln \rho\left(x_{+\tau}\right)-\beta Q[x(t), \lambda(t)] .
$$

This is the change in the amount of information required to describe the microscopic state of the system plus the change in entropy of the bath. 
Recall that the fluctuation theorem, Eq. (2), compares the entropy production probability distribution of a process with the entropy production distribution of the corresponding time-reversed process. For example, with the confined gas we compare the entropy production when the gas is compressed to the entropy production when the gas is expanded. To allow this comparison of forward and reverse processes, we will require that the entropy production is odd under a time reversal, i.e., $\omega_{\mathrm{F}}=-\omega_{\mathrm{R}}$, for the process under consideration. This condition is equivalent to requiring that the final distribution of the forward process, $\rho_{\mathrm{F}}\left(x_{+\tau}\right)$, is the same (after a time reversal) as the initial phase-space distribution of the reverse process, $\rho_{\mathrm{R}}\left(\bar{x}_{+\tau}\right)$, and vice versa, i.e., $\rho_{\mathrm{F}}\left(x_{+\tau}\right)=\rho_{\mathrm{R}}\left(\bar{x}_{+\tau}\right)$ and $\rho_{\mathrm{R}}\left(x_{-\tau}\right)=\rho_{\mathrm{F}}\left(\bar{x}_{-\tau}\right)$. In the next section, we will discuss two broad types of work process that fulfill this condition. Either the system begins and ends in equilibrium or the system begins and ends in the same time symmetric nonequilibrium steady state.

This time-reversal symmetry of the entropy production allows the comparison of the probability of a particular path, $x(t)$, starting from some specific point in the initial distribution, with the corresponding time-reversed path,

$$
\frac{\rho_{\mathrm{F}}\left(x_{-\tau}\right) \mathcal{P}[x(+t) \mid \lambda(+t)]}{\rho_{\mathrm{R}}\left(\bar{x}_{+\tau}\right) \mathcal{P}[\bar{x}(-t) \mid \bar{\lambda}(-t)]}=e^{+\omega_{\mathrm{F}}} .
$$

This follows from the the conditions that the system is microscopically reversible, Eq. (5), and that the entropy production is odd under a time reversal.

Now consider the probability, $P_{\mathrm{F}}(\omega)$, of observing a particular value of this entropy production. It can be written as a $\delta$ function averaged over the ensemble of forward paths,

$$
\begin{aligned}
P_{\mathrm{F}}(\omega)= & \left\langle\delta\left(\omega-\omega_{\mathrm{F}}\right)\right\rangle_{\mathrm{F}} \\
= & \iiint_{x_{-\tau}}^{x_{+\tau}} \rho_{\mathrm{F}}\left(x_{-\tau}\right) \mathcal{P}[x(+t) \mid \lambda(+t)] \\
& \times \delta\left(\omega-\omega_{\mathrm{F}}\right) \mathcal{D}[x(t)] d x_{-\tau} d x_{+\tau} .
\end{aligned}
$$

Here $\iiint_{x_{-\tau}}^{x_{+\tau}} \ldots \mathcal{D}[x(t)] d x_{-\tau} d x_{+\tau}$ indicates a sum or suitable normalized integral over all paths through phase-space, and all initial and final phase-space points, over the appropriate time interval. We can now use Eq. (7) to convert this average over forward paths into an average over reverse paths,

$$
\begin{aligned}
P_{\mathrm{F}}(\omega)= & \iiint_{x_{-\tau}}^{x_{+\tau}} \rho_{\mathrm{R}}\left(\bar{x}_{+\tau}\right) \mathcal{P}[\bar{x}(-t) \mid \bar{\lambda}(-t)] \\
& \times \delta\left(\omega-\omega_{\mathrm{F}}\right) e^{+\omega_{\mathrm{F}}} \mathcal{D}[x(t)] d x_{-\tau} d x_{+\tau} \\
= & e^{+\omega} \iiint_{x_{-\tau}}^{x_{+\tau}} \rho_{\mathrm{R}}\left(\bar{x}_{+\tau}\right) \mathcal{P}[\bar{x}(-t) \mid \bar{\lambda}(-t)] \\
& \times \delta\left(\omega+\omega_{\mathrm{R}}\right) \mathcal{D}[x(t)] d x_{-\tau} d x_{+\tau} \\
= & e^{+\omega}\left\langle\delta\left(\omega+\omega_{\mathrm{R}}\right)\right\rangle_{\mathrm{R}}=e^{+\omega} P_{\mathrm{R}}(-\omega) .
\end{aligned}
$$

The $\delta$ function allows the $e^{+\omega}$ term to be moved outside the integral in the second line above. The remaining average is over reverse paths as the system is driven in reverse. The final result is the entropy production fluctuation theorem, Eq. (2).

The theorem readily generalizes to other ensembles. As an example, consider an isothermal-isobaric system. In addition to the heat bath, the system is coupled to a volume bath, characterized by $\beta p$, where $p$ is the pressure. Then the microscopically reversible condition, Eq. (5), becomes

$$
\begin{aligned}
\frac{\mathcal{P}[x(+t) \mid \lambda(+t)]}{\mathcal{P}[\bar{x}(-t) \mid \bar{\lambda}(-t)]}= & \exp \{-\beta Q[x(t), \lambda(t)] \\
& -\beta p \Delta V[x(t), \lambda(t)]\} .
\end{aligned}
$$

Both baths are considered to be large, equilibrium, thermodynamic systems. Therefore, the change in entropy of the heat bath is $-\beta Q$ and the change in entropy of the volume bath is $-\beta p \Delta V$, where $\Delta V$ is the change in volume of the system. The entropy production should then be defined as

$$
\omega=\ln \rho\left(x_{-\tau}\right)-\ln \rho\left(x_{+\tau}\right)-\beta Q-\beta p \Delta V .
$$

The fluctuation theorem, Eq. (2), follows as before. It is possible to extend the fluctuation theorem to any standard set of baths, so long as the definitions of microscopic reversibility and the entropy production are consistent. In the rest of this paper we shall only explicitly deal with systems coupled to a single heat bath, but the results generalize directly.

\section{TWO GROUPS OF APPLICABLE SYSTEMS}

In this section we will discuss two groups of systems for which the entropy fluctuation theorem, Eq. (2), is valid. These systems must satisfy the condition that the entropy production, Eq. (6), is odd under a time reversal, and therefore that $\rho_{\mathrm{F}}\left(x_{+\tau}\right)=\rho_{\mathrm{R}}\left(\bar{x}_{+\tau}\right)$.

First consider a system that is in equilibrium from time $t=-\infty$ to $t=-\tau$. It is then driven from equilibrium by a change in the controlled parameter, $\lambda$. The system is actively perturbed up to a time $t=+\tau$, and is then allowed to relax, so that it once again reaches equilibrium at $t=+\infty$. For the forward process the system starts in the equilibrium ensemble specified by $\lambda(-\infty)$, and ends in the ensemble specified by $\lambda(+\infty)$. In the reverse process, the initial and final ensembles are exchanged, and the entropy production is odd under this time reversal. The gas confined in the diathermal cylinder satisfies these conditions if the piston moves only for a finite amount of time.

At first it may appear disadvantageous that the entropy production has been defined between equilibrium ensembles separated by an infinite amount of time. However, for these systems the entropy production has a simple and direct interpretation. The probability distributions of the initial and final ensembles are known from equilibrium statistical mechanics:

$$
\rho_{\mathrm{eq}}(x \mid \lambda)=\frac{e^{-\beta E(x, \lambda)}}{\sum_{x} e^{-\beta E(x, \lambda)}}=\exp \{\beta F(\beta, \lambda)-\beta E(x, \lambda)\}
$$




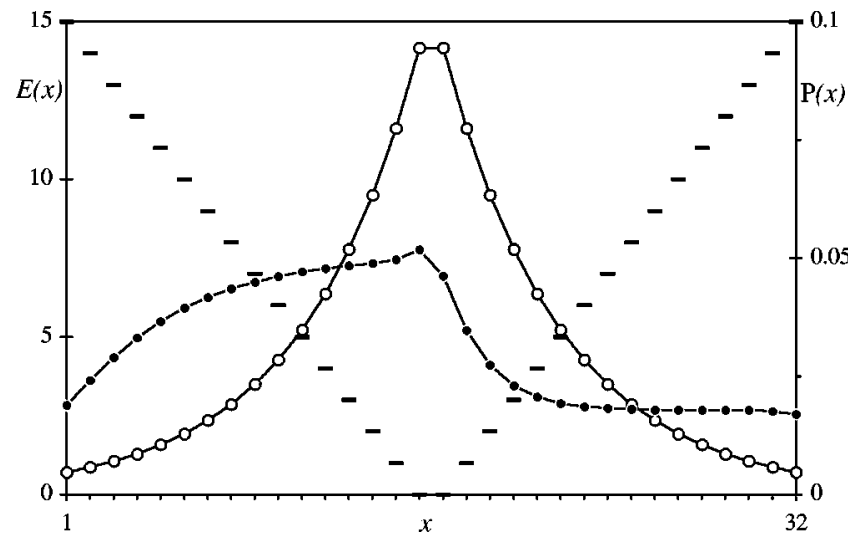

FIG. 1. A very simple Metropolis Monte Carlo simulation is used to illustrate the fluctuation theorem and some of its approximations. The master equation for this system can be solved, providing exact numerical results to compare with the theory. A single particle occupies a finite number of positions in a one-dimensional box with periodic boundaries, and is coupled to a heat bath of temperature $T=5$. The energy surface, $E(x)$, is indicated by - in the figure. At each discrete time step the particle attempts to move left, right, or stay put with equal probability. The move is accepted with the standard Metropolis acceptance probability [34]. Every eight time steps, the energy surface moves right one position. Thus the system is driven away from equilibrium, and eventually settles into a time symmetric nonequilibrium steady state. The equilibrium $(\bigcirc)$ and steady-state $(O)$ probability distributions are shown in the figure above. The steady-state distribution is shown in the reference frame of the surface.

The sum is over all states of the system, and $F(\beta, \lambda)=$ $-\beta^{-1} \ln \Sigma_{x} \exp \{-\beta E(x, \lambda)\}$ is the Helmholtz free energy of the system. If these probabilities are substituted into the definition of the entropy production, Eq. (6), then we find that

$$
\omega_{\mathrm{F}}=-\beta \Delta F+\beta W
$$

Here $W$ is the work and we have used the first law of thermodynamics, $\Delta E=W+Q$.

It is therefore possible to express the fluctuation theorem in terms of the amount of work performed on a system that starts in equilibrium [30],

$$
\frac{P_{\mathrm{F}}(+\beta W)}{P_{\mathrm{R}}(-\beta W)}=e^{-\Delta F} e^{+\beta W} .
$$

The work in this expression is measured over the finite time that the system is actively perturbed. We have now established the fluctuation theorem for systems that start in equilibrium, and have shown that the entropy production is simply related to the work and free energy change. Therefore, we have established the nonequilibrium work relation, Eq. (3), discussed in the Introduction.

The validity of this expression can be illustrated with the very simple computer model described in Fig. 1. Although not of immediate physical relevance, this model has the advantage that the entropy production distributions of this driven nonequilibrium system can be calculated exactly, apart from numerical roundoff error. The resulting work distributions are shown in Fig. 2. Because the process is time symmetric, $\Delta F=0$ and $P(+\beta W)=P(-\beta W) \exp \beta W$. This

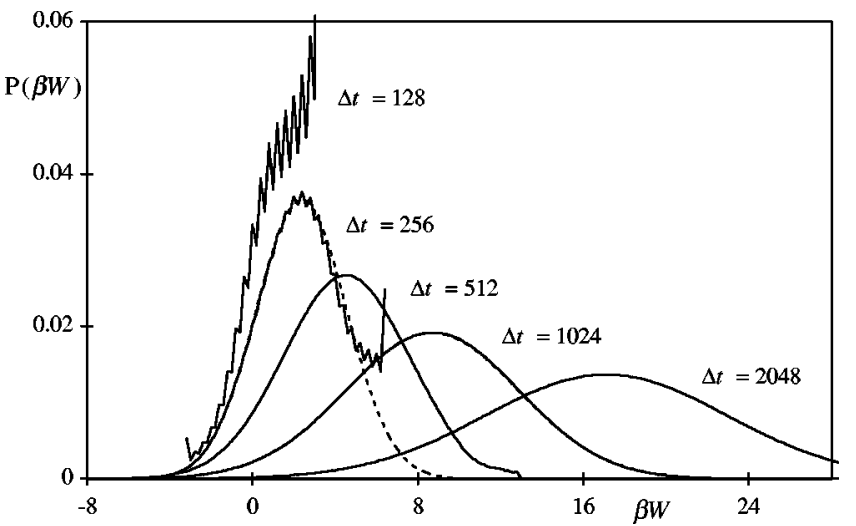

FIG. 2. Work probability distribution (-) for the system of Fig. 1 , starting from equilibrium. The work $W$ was measured over 16 , $32,64,128$, and 256 cycles $(\Delta t=128,256,512,1024$, and 2048). For each of these distributions the work fluctuation theorem, Eq. (11), is exact. The dashed lines (- - -) are Gaussians fitted to the means of the distributions for $\Delta t=256$ and 1024. (See Sec. IV.)

expression is exact for each of the distributions shown, even for short times when the distributions display very erratic behavior.

Systems that start and end in equilibrium are not the only ones that satisfy the fluctuation theorem. Consider again the classical gas confined in a diathermal cylinder. If the piston is driven in a time symmetric periodic manner (for example, the displacement of the piston is a sinusoidal function of time), the system will eventually settle into a nonequilibrium steady-state ensemble. We will now require that the dynamics are entirely diffusive, so that there are no momenta. Then at any time that $\lambda(t)$ is time symmetric, then the entire system is invariant to a time reversal. We start from the appropriate nonequilibrium steady state, at a time symmetric point of $\lambda(t)$, and propagate forward in time a whole number of cycles. The corresponding time-reversed process is then identical to the forward process, with both starting and ending in the same steady-state ensemble. The entropy production for this system is odd under a time reversal and the fluctuation theorem is valid.

As a second example, consider a fluid under a constant shear [1]. The fluid is contained between parallel walls which move relative to one another, so that $\lambda(t)$ represents the displacement of the walls. Eventually the system settles into a nonequilibrium steady state. A time reversal of this steady-state ensemble will reverse all the velocities, including the velocity of the walls. The resulting ensemble is related to the original one by a simple reflection, and is therefore effectively invariant to the time reversal. Again, the forward process is identical to the reverse process, and the entropy production is odd under a time reversal.

In general, consider a system driven by a time symmetric, periodic process. $[\lambda(t)$ is a periodic, even function of time.] We require that the resulting nonequilibrium steady-state ensemble be invariant under time reversal, assuming that we pick a time about which $\lambda(t)$ is also symmetric. This symmetry ensures that the the forward and reverse processes are essentially indistinguishable, and therefore that the entropy production is odd under a time reversal. It is no longer necessary to explicitly label forward and reverse processes. $P_{\mathrm{F}}(\omega)=P_{\mathrm{R}}(\omega)=P(\omega)$. For these time symmetric steady- 


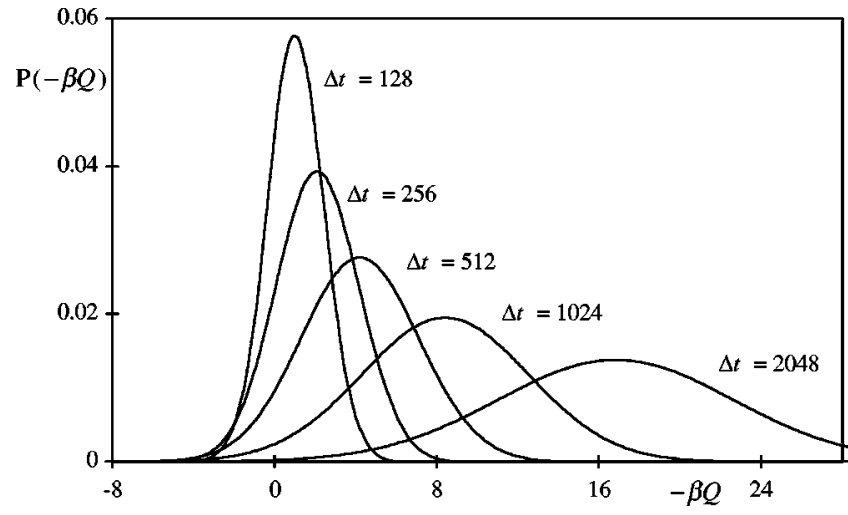

FIG. 3. Heat probability distribution (-) for the nonequilibrium steady state. The model described in Fig. 1 was relaxed to the steady state, and the heat $Q$ was then measured over $16,32,64,128$, and 256 cycles $(\Delta t=128,256,512,1024$, and 2048). Note that for long times the system forgets its initial state and the heat distribution is almost indistinguishable from the work distribution of the system that starts in equilibrium.

state ensembles the fluctuation theorem is valid for any integer number of cycles, and can be expressed as

$$
\frac{P(+\omega)}{P(-\omega)}=e^{+\omega} .
$$

For a system under a constant perturbation (such as the sheared fluid) this relation is valid for any finite time interval.

\section{LONG TIME APPROXIMATIONS}

The steady-state fluctuation theorem, Eq. (12), is formally exact for any integer number of cycles, but is of little practical use because, unlike the equilibrium case, we have no independent method for calculating the probability of a state in a nonequilibrium ensemble. The entropy production is not an easily measured quantity. However, we can make a useful approximation for these nonequilibrium systems which is valid when the entropy production is measured over long time intervals.

From the relation $\langle\exp (-\omega)\rangle=1$, Eq. (4), and the inequality $\langle\exp x\rangle \geqslant \exp \langle x\rangle$, which follows from the convexity of $e^{x}$, we can conclude that $\langle\omega\rangle \geqslant 0$. On average the entropy production is positive. Because the system begins and ends in the same probability distribution, the average entropy production depends only on the average amount of heat transferred to the bath. $-\langle\omega\rangle=\langle\beta Q\rangle \leqslant 0$. On average over each cycle, energy is transferred through the system and into the heat bath (Clausius inequality). The total heat transferred tends to increase with each successive cycle. When measurements are made over many cycles, the entropy production will be dominated by this heat transfer and $\omega \approx-\beta Q$. Therefore, in the long time limit the steady-state fluctuation theorem, Eq. (12), can be approximated as

$$
\lim _{\Delta t \rightarrow \infty} \frac{P(+\beta Q)}{P(-\beta Q)}=\exp (\beta Q) .
$$

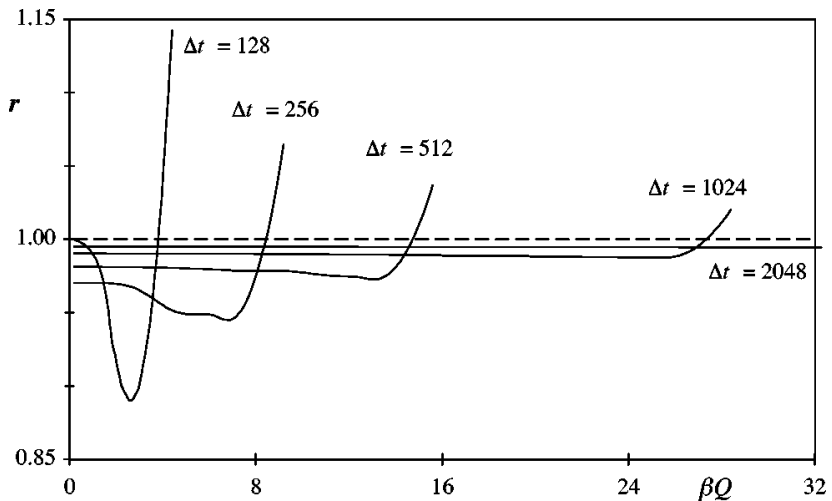

FIG. 4. Deviations from the heat fluctuation theorem, Eq. (13), for the distributions of Fig. 3. If the heat fluctuation theorem were exact, then the ratio $r=\beta Q / \ln [P(+\beta Q) / P(-\beta Q)]$ would equal 1 for all $|\beta Q|$. For short times $(\Delta t \leqslant 256)$ the fluctuation theorem is wholly inaccurate. For times significantly longer than the relaxation time of the system $(\Delta t \approx 100)$, the fluctuation theorem is accurate except for very large values of $|\beta Q|$.

Because $-\beta Q$ is the change in entropy of the bath, this heat fluctuation theorem simply ignores the relatively small and difficult to measure microscopic entropy of the system.

Heat distributions for the simple computer model of a nonequilibrium steady state are shown in Fig. 3, and the validity of the above approximation is shown in Fig. 4. As expected, the heat fluctuation theorem, Eq. (13), is accurate for times much longer than the relaxation time of the system.

Another approximation to the entropy production probability distribution can be made in this long time limit. For long times the entropy production $\omega$ is the sum of many weakly correlated values and its distribution should be approximately Gaussian by the central limit theorem. If the driving process is time symmetric, then $P_{\mathrm{F}}(\omega)=P_{\mathrm{R}}(\omega)$ $=P(\omega)$, and the entropy production distribution is further constrained by the fluctuation theorem itself. The only Gaussians that satisfy the fluctuation theorem have a variance twice the mean, $2\langle\omega\rangle=\left\langle(\omega-\langle\omega\rangle)^{2}\right\rangle$. This is a version of the standard fluctuation-dissipation relation [31]. The mean en-

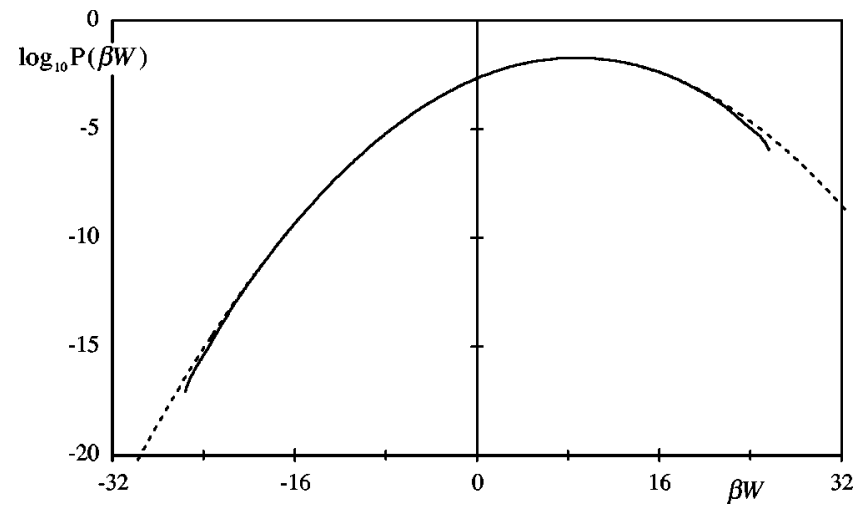

FIG. 5. Work distribution (-) and Gaussian approximation (- - ) for $\Delta t=2048$, with the probabilities plotted on a logarithmic scale. The Gaussian is fitted to the mean of the distribution and has a variance half the mean (see Sec. IV). This Gaussian approximation is very accurate, even to the very wings of the distribution, for times much longer than the relaxation time $(\Delta t \approx 100)$ of the system. This same distribution is shown on a linear scale in Fig. 2. 
tropy production (dissipation) is related to the fluctuations in the entropy production. If these distributions are Gaussian, then the fluctuation theorem implies the Green-Kubo relations for transport coefficients $[1,32,33]$. However, we have not used the standard assumption that the system is close to equilibrium. Instead, we have assumed that the system is driven by a time symmetric process, and that the entropy production is measured over a long time period.

Gaussian approximations are shown in Figs. 2 and 5 for the work distribution of the simple computer model. For times much longer than the relaxation time of the system, these approximations are very accurate, even in the wings of the distributions. This is presumably due to the symmetry imposed by the fluctuation theorem. For a nonsymmetric driving process, this symmetry is broken, and the distributions will not necessarily satisfy the fluctuation-dissipation relation in the long time limit. For example, see Fig. 8 of Ref. [16]. Clearly these distributions will be poorly approximated by the Gaussian distributions considered here.

\section{SUMMARY}

The fluctuation theorem, Eq. (2), appears to be very general. In this paper we have derived a version that is exact for finite time intervals, and which depends on the following assumptions; the system is finite and classical, and coupled to a set of baths, each characterized by a constant intensive parameter. The dynamics are required to be stochastic, Markovian, and microscopically reversible, Eq. (5), and the entropy production, defined by Eq. (6), must be odd under a time reversal. This final condition is shown to hold for two broad classes of systems, those that start in equilibrium and those in a time symmetric nonequilibrium steady state. For the latter systems the fluctuation theorem holds for entropy productions measured over any integer number of cycles. This generality, and the existence of the nonequilibrium work relation discussed in the Introduction, suggests that other nontrivial consequences of the fluctuation theorem are awaiting study.

\section{ACKNOWLEDGMENTS}

This work was initiated with support from the National Science Foundation under Grant No. CHE-9508336, and completed with support from the U.S. Department of Energy under Contract No. DE-AC03-76SF00098. I am indebted to C. Jarzynski and C. Dellago for their helpful discussions, and D. Chandler for his advice and guidance.
[1] D. J. Evans, E. G. D. Cohen, and G. P. Morriss, Phys. Rev. Lett. 71, 2401 (1993).

[2] D. Evans and D. Searles, Phys. Rev. E 50, 1645 (1994).

[3] G. Gallavotti and E. G. D. Cohen, Phys. Rev. Lett. 74, 2694 (1995).

[4] G. Gallavotti and E. G. D. Cohen, J. Stat. Phys. 80, 931 (1995).

[5] D. Evans and D. Searles, Phys. Rev. E 53, 5808 (1996).

[6] G. Gallavotti, J. Stat. Phys. 84, 899 (1996).

[7] E. G. D. Cohen, Physica A 240, 43 (1997).

[8] G. Gallavotti, Chaos 8, 384 (1998).

[9] J. Kurchan, J. Phys. A 31, 3719 (1998).

[10] D. Ruelle, J. Stat. Phys. 95, 393 (1999); e-print mp-arc/98-770.

[11] J. L. Lebowitz and H. Spohn, J. Stat. Phys. 95, 333 (1999); e-print cond-mat/9811220.

[12] C. Maes, J. Stat. Phys. 95, 367 (1999); e-print mp-arc/98-754.

[13] E. Cohen and G. Gallavotti, e-print cond-mat/9903418.

[14] G. Ayton and D. Evans, e-print cond-mat/9903409.

[15] C. Jarzynski, Phys. Rev. Lett. 78, 2690 (1997).

[16] C. Jarzynski, Phys. Rev. E 56, 5018 (1997).

[17] C. Jarzynski, Acta Phys. Pol. B 29, 1609 (1998).

[18] C. Jarzynski, e-print cond-mat/9802249.

[19] G. E. Crooks, J. Stat. Phys. 90, 1481 (1998).
[20] C. H. Bennett, J. Comput. Phys. 22, 245 (1976).

[21] D. Chandler, Introduction to Modern Statistical Mechanics (Oxford University Press, New York, 1987).

[22] T. P. Straatsma and J. A. McCammon, Annu. Rev. Phys. Chem. 43, 407 (1992).

[23] D. Frenkel and B. Smit, Understanding Molecular Simulation: From Algorithms to Applications (Academic Press, San Diego, 1996).

[24] C. E. Shannon, Bell Syst. Tech. J. 27, 379 (1948).

[25] G. R. Grimmett and D. R. Stirzaker, Probability and Random Processes, 2nd ed. (Clarendon Press, Oxford, 1992).

[26] S. R. de Groot and P. Mazur, Non-equilibrium Thermodynamics (North-Holland, Amsterdam, 1962).

[27] R. C. Tolman, Phys. Rev. 23, 693 (1924).

[28] R. C. Tolman, The Principles of Statistical Mechanics (Oxford University Press, London, 1938), pp. 163 and 165.

[29] This definition of the entropy production is equivalent to the quantity $\Sigma$ used by Jarzynski [16].

[30] C. Jarzynski (private communication).

[31] H. B. Callen and T. A. Welton, Phys. Rev. 83, 34 (1951).

[32] D. Evans and D. Searles, Phys. Rev. E 52, 5839 (1995).

[33] D. J. Searles and D. J. Evans, e-print cond-mat/9902021.

[34] N. Metropolis et al., J. Chem. Phys. 21, 1087 (1953). 